\title{
The Care of HIV-Infected Persons: A Specialty?
}

The worldwide epidemic of acquired immunodeficiency syndrome (AIDS) and the treatment of human immunodeficiency virus (HIV) infection has for the past 15 years been the most rapidly evolving area in medical care. Early in the epidemic, when the number of AIDS cases was growing rapidly, it was proposed that every primary care clinician would need to manage HIV-infected persons, and many family physicians stepped into the forefront of HIV care. Now there are concerns that the care of HIV-infected persons has grown extremely complex and calls from some quarters for the development of specialists in HIV medicine. ${ }^{1-3}$

In this issue of the fournal, William Brandon ${ }^{4}$ surveys the impact of the growing complexity of HIV treatment on the attitudes and practices of family physicians attending a HIV continuing education workshop at the 1996 American Academy of Family Physicians Scientific Assembly. He finds that these family physicians are comfortable caring for HIV-infected persons and expect to continue to do so. It should be remembered that the group surveyed was attending a continuing medical education update on HIV infection and probably does not reflect all family physicians; more likely this group reflects those family physicians who provide care to HIV-infected persons. He also suggests that there might eventually develop criteria whereby someone is certified as an expert in HIV care and calls on the professional organizations within family practice to take an active role in ensuring that family physicians are included should this occur.

As a family physician whose practice is limited to persons with HIV infection, I have long been interested in this issue. It appears to me that, on the surface, there are two questions: should there be a specialty in the care of HIV-infected persons, and if there is a specialty, where will the specialists come from? I believe that both these questions

Submitted 24 November 1998.

From a private practice, Tucson, Ariz. Address reprint requests to J. Kevin Carmichael, MD, El Rio Special Immunology Associates, 445 North Silverbell, Suite 202, Tucson, AZ 85745 . have been answered by physicians and their patients. It is clear to me there already are HIV specialists, and they have come from every field of medicine. The real question now lies in how we recognize and organize what has already occurred to ensure optimal care for persons living with HIV infection.

The specialty of HIV medicine has evolved from the nature of the HIV epidemic itself. Early on, the populations affected, the uncertainty about the cause, the lack of effective therapy, and the high death rate kept HIV infection from becoming the responsibility of any single existing specialty. These characteristics attracted those providers who found in HIV-infected patient care something that resonated within. It was a disease that (almost) no provider wanted to care for. Those providers who were willing and able to do the work were rapidly sought out by HIV-infected patients, developed reputations in their communities, and found their practices becoming primarily the care of persons living with HIV infection.

I realized I had become this kind of specialist the day I saw myself on television with the caption "AIDS Doctor" underneath. Those early years, before effective antiretroviral therapy, while horrific ( $\mathrm{I}$ had 76 patients die in 1 year), were also fantastically rewarding. The magnitude of the crisis dwarfed all other concerns. The health care system really did not know how to deal with HIV infection, and thus HIV care providers generally had freedom to work with their patients and the social and political organizations that developed to battle this illness. I still have a cartoon of myself as a muscle-bound warrior, wielding a battle-ax and entwined in combat with a fanged serpent, titled "Dr. Carmichael battles AIDS" drawn by a patient and given to me on the day he died.

More recently the advent of highly active antiretroviral therapy (HAART) has dramatically altered the course of HIV disease. The death rate in my practice has declined from 14 in 100 patient years in 1995 to 2 in 100 patient years in 1998 . The complexity of this therapy and its continued rapid evolution requires a near-daily reassessment of what constitutes optimal therapy. Despite ex- 
tensive published guidelines, decisions must be made daily about situations outside these guidelines and in the absence of any published data. This complexity, the realization that inadequate treatment can promote the development of drug resistance and drug failure, and a growing recognition of the important toxicities of HAART have added weight to the argument for specialization. Additionally, data have accrued to support the notion that experienced providers have better outcomes when treating opportunistic infections. ${ }^{3}$ Bruce Soloway, ${ }^{5}$ however, has noted that there are no data suggesting quality of care or patient outcome in HIV treatment is dependent on the field in which the physician received his or her formal training.

Economic factors too have driven some to call for the development of a specialty in HIV medicine. HIV infection is a complex and expensive disease. The bulk of the cost of HIV care is increasingly falling to health maintenance organizations, Medicaid, and the Ryan White programs. Successful HAART has resulted in a decrease in the overall costs of HIV care by decreasing the incidence of opportunistic infection and the amount of inpatient care. There has been a shift of cost toward outpatient care, with medications and laboratory testing responsible for the largest proportion of these costs. Many HIV care providers are finding that capitated systems do not recognize or reward the increased complexity of HIV care. Some managed care plans discriminate against experienced HIV care providers to avoid adverse selection of their plans by persons living with HIV. Some feel that the solution to this problem is to develop a specialty and thereby force an acknowledgment of and compensation for the added complexity of HIV medicine.

If HIV specialists already exist and have come from every field of medicine, then how are they to be recognized and defined? It is perhaps simplest to define experts by the work they do. HIV specialists care for persons with HIV infection. HIV medicine, though perhaps becoming a specialty, is a quintessential primary care disease. HIV has an impact on every organ system and every aspect of the patient's life. The majority of HIV specialists practice primary care, that is, they accept responsibility for managing all of the problems the patient experiences. HIV specialists who have come from primary care fields such as family practice and gen- eral internal medicine have had to learn virology, immunology, and pharmacokinetics among many other subspecialty areas. HIV specialists who have come from specialty fields have had to learn how to address the psychosocial dimensions of HIV, how to do Papanicolaou smears and skin biopsies, how to work with case managers and patient advocates, and how to provide primary care.

There are clearly risks to the development of a specialty in HIV medicine. Specialties are by definition exclusive and exclusionary. We should view with suspicion any plan growing out of the most stigmatized illness of our time that will exclude physicians from providing care. Some argue there already exists within HIV care a two-tiered system largely based on socioeconomic status and fear that a specialty designation will only solidify this split. Those who can get access to specialists will do so, but those who cannot will be left behind as fewer educational and other resources are devoted toward nonspecialists. The HIV epidemic continues to evolve rapidly, shifting toward lower socioeconomic classes and toward rural areas. The Ryan White programs currently support large portions of the HIV-specific care delivered to the uninsured. Will these programs continue if the political voice of the AIDS epidemic fades, and the sense of crisis wanes? And will there then be HIV medicine specialists outside large cities and large universities?

HIV care has remained remarkably free of turf battles largely because of the enormous challenge it presents and because those caring for infected patients have always used all the help we could get. Much like the wisdom of the scarecrow, the courage of the lion, and the compassion of the tin man in the Wizard of $\mathrm{Oz}$, the development of a HIV medicine specialty has occurred but remains to be formally organized. Many HIV caregivers feel the time has come to develop a professional home. The Infectious Disease Society of America has recently appointed a committee of HIV experts from internal medicine, pediatrics, obstetrics and gynecology, and family practice to define the parameters of HIV medicine and consider the means by which HIV medicine specialists might be developed and recognized. Ideally, a professional home for HIV medicine specialists will recognize the contributions of all specialties to the care of HIV-infected persons and will be stronger and more successful as a result of the variety of tal- 
ents brought from all the medical specialties. I hope HIV medicine specialists have learned well the lessons AIDS has taught thus far and will continue to advocate in both the political and medical arena for the optimal care of persons living with HIV infection.

\section{J. Kevin Carmichael, MD \\ Tucson, Ariz}

\section{References}

1. Volberding PA. Should the treatment of HIV infection be left to specialists? HIV Newsline 1998;4:51-5.
2. Zuger A, Sharp VL. "HIV specialists": the time has come. JAMA 1997;278:1131-2.

3. Kitihata MM, Koepsell TD, Deyo RA, Maxwell CL, Dodge WT, Wagner EH. Physicians' experience with the acquired immunodeficiency syndrome as a factor in patients' survival. N Engl J Med 1996;334: 701-6.

4. Brandon W. Changing treatment regimens for HIV infection: impact on referral patterns and training needs of family physicians. J Am Board Fam Pract 1999;12:000-00.

5. Soloway B. Primary care and specialty care in the age of HAART. AIDS Clin Care 1997;9:37-9. 